\title{
A LOSSLESS COLOR IMAGE COMPRESSION USING AN IMPROVED REVERSIBLE COLOR TRANSFORM WITH HIERARCHICAL PREDICTION AND MODIFIED HUFFMAN CODING
}

\author{
Priyatosh Halder', Suman ${ }^{2}$ \\ ${ }^{1}$ Radar Section, Air Force Jodhpur, Rajasthan, India \\ ${ }^{2}$ Asst Professor, ECE Department, Sri Sukhmani Institute of Engineering \& Technology, Dera Bassi, Punjab
}

\begin{abstract}
In case of the conventional lossless color image compression methods, the pixels are interleaved from each color component, and they are predicted and finally encoded. In this paper, we propose a lossless color image compression method using hierarchical prediction of chrominance channel pixels and encoded with modified Huffman coding. An input image is chosen and the $R, G$ and $B$ color channel is transform into $\mathrm{YCuCv}$ color space using an improved reversible color transform. After that a conventional lossless image coder like CALIC is used to compress the luminance channel $Y$. The chrominance channel $\mathrm{Cu}$ and $\mathrm{Cv}$ are encoded with hierarchical decomposition and directional prediction. The effective context modeling for prediction residual is adopted finally. It is seen from the experimental result the proposed method improves the compression performance than the existing method.
\end{abstract}

Keywords: Lossless color image compression, hierarchical prediction, reversible color transform, modified Huffman coding.

\section{INTRODUCTION}

Recently increase in image related applications have created an issue of the transformation of the images and its storing. The considerable amount of space and bandwidth is required for storage and transmission of image. This problem can be overcome by the image compression. It reduces the number of bites required to represent an image. So, the image compression is an important part in the research field. Recently, there are a number of image compression methods are available. These can be classified into two types: lossless and lossy compression.

In lossless compression, the reconstructed image is exactly same as compressed image. So, it is used in various type of images like medical, scientific and artistic image. There are so many lossless image compression algorithms are available. The algorithms which are widely used are JPEGLS [1], lossless JPEG [2], Low Complexity Lossless Compression for images [3], Context based Adaptive Lossless Image Codec [4], JPEG XR [5] and JPEG2000 [6]. The CALIC and LOCO-I were developed for the JPEG standardization, whereas almost similarity is there between the CALIC and LOCO-I but the CALIC provide better compression rate at the more computational complexity. The most of the conventional algorithm were developed for grayscale image. Some modifications were carried out for the compression of hyper-spectral or color images [7, 8]. In this paper, we have been proposed a lossless color image compression algorithm that efficiently compresses the chrominance channels by using an improved RCT $[9,10]$ and the hierarchical prediction $[11,12]$ of the pixels of chrominance channel. After the RCT, the compression of the luminance channel $\mathrm{Y}$ is done by a conventional lossless image coder, such as CALIC. With the help of new methods the chrominance channel $\mathrm{Cu}$ and $\mathrm{Cv}$ are compressed. The generated prediction errors from the chrominance channels are small in the smooth region but relatively large near the edge or texture region. To get the accurate prediction, we decompose a chrominance image into two subimages, one of which consists of even numbered of rows from the input image and the other odd. Then, the even subimage Xe is used to predict the odd subimage. In two stages this decomposition and prediction is carried out. The directional predictor is employed to reduce large prediction error near the object edges. Lastly, for the prediction error the effective context modeling is adopted, which gets information from neighboring pixels.

The paper can be divided as: section II describes the improved reversible color transform (RCT). And the hierarchical decomposition and detailed prediction process is represented by section III. The condition of the proposed work has given in section IV. The section $\mathrm{V}$ shows the experimental results. Finally, we share the conclusion of the work in the section VI.

\section{IMPROVED REVERSIBLE COLOR}

\section{TRANSFORM}

Usually per pixel the three color values: red, green and blue are used to represent the natural image. Due to the significant correlation of color channels with each other, a 
color transform is used before compression. For example, for lossy compression the popular YCbCr transform used is defined as below. But due to not exact invertible transform with integer arithmetic, it cannot be used for lossless image compression.

$\left(\begin{array}{l}Y \\ C_{b} \\ C_{r}\end{array}\right)=\left(\begin{array}{lll}0.299 & 0.587 & 0.114 \\ -0.16875 & -0.33126 & 0.5 \\ 0.5 & -0.41869 & -0.08131\end{array}\right)\left(\begin{array}{l}R \\ G \\ B\end{array}\right)$

In case of the JPEG 2000, a reversible color transform $(\mathrm{RCT})$ is introduced as follows:

$$
\begin{array}{ccc}
\mathrm{Y}=(\mathrm{R}+2 \mathrm{G}+\mathrm{B}) / 4 & \mathrm{G}=\mathrm{Y}-(\mathrm{Cu}+\mathrm{Cv}) / 4 \\
\mathrm{Cu}=\mathrm{R}-\mathrm{G} & \leftrightarrow & \mathrm{R}=\mathrm{Cu}+\mathrm{G} \\
\mathrm{Cv}=\mathrm{B}-\mathrm{G} \quad \leftrightarrow \quad \mathrm{B}=\mathrm{Cv}+\mathrm{G}
\end{array}
$$

This is a simple transform. This transformation is known as "reversible" transform because it is exactly invertible with integer arithmetic. But the satisfactory performance of decorrelation does not provided by it.

In case of improved RCT for increasing the decorrelation performance, a lifting process is added after the RCT as shown in Fig. 1.

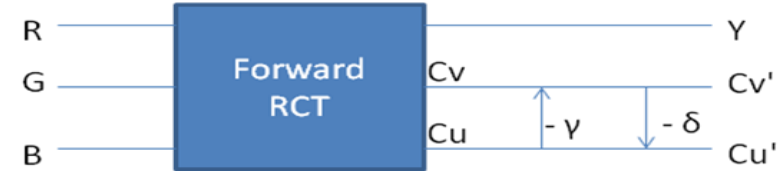

Fig - 1: Flow graph of the improved RCT

It gives the output as follow:

$$
\begin{gathered}
\mathrm{Cv}^{\prime}=\mathrm{Cv}-[\gamma \mathrm{Cu}] \\
\mathrm{Cu}^{\prime}=\mathrm{Cu}-\left[\delta \mathrm{Cv}^{\prime}\right]
\end{gathered}
$$

Where $\gamma$ and $\delta$ are scalar gains for prediction and update steps in the lifting butterfly. Here for the existence of inverse transform the floor operations on $[\gamma \mathrm{Cu}]$ and $[\delta \mathrm{Cv}]$ are required. Another expression of (3) without floor function is as given below:

$$
\left(\begin{array}{l}
\mathrm{Cv}^{\prime} \\
\mathrm{Cu}
\end{array}\right) \approx\left(\begin{array}{lll}
-\gamma & -1+\gamma & 1 \\
1+\gamma \delta & -1-\delta+\gamma \delta & -\delta
\end{array}\right)\left(\begin{array}{l}
\mathrm{R} \\
\mathrm{G} \\
\mathrm{B}
\end{array}\right)
$$

Since the transform of (1) performs better than simple RCT of (2), so in case of improved RCT the two parameters $\gamma$ and $\delta$ is find out such a way it approximates (1) while keeping the reversibility. By comparing (4) and (1), we get the value of $\gamma$ and $\delta$ are $2 \times 0.16875$ and $2 \times 0.08131$ respectively which may be the reasonable choice.

\section{HIERARCHICAL DECOMPOSITION AND PIXEL PREDICTION}

The chrominance channels $\mathrm{Cu}$ and $\mathrm{Cv}$ which is available after the reversible color transform have some different statistical characteristics from Luminance $\mathrm{Y}$ or original color planes R, G and B. The chrominance channel, through the color transform the signal variation is suppressed, but near the object boundary it still remains. As a result, in the smooth region the prediction error of the chrominance channels $\mathrm{Cu}$ and $\mathrm{Cv}$ are much reduced but near the edge and texture it remains comparably larger. So along with the exact prediction the importance is given to estimate the accurate magnitude of prediction error.

For this purpose, a hierarchical decomposition scheme is employed. In Fig. 2, all the pixels in the input image $\mathrm{A}$ is separated into two subimages: an odd subimage $\mathrm{A}_{\mathrm{o}}$ and an even subimage $A_{e}$. The $A_{o}$ is predicted by the $A_{e}$. This decomposition and prediction is performed into two levels.

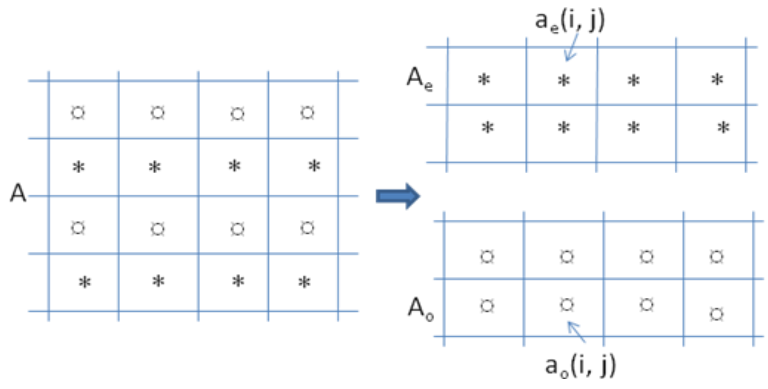

Fig - 2: Input images and its decomposition subimages

To avoid the large prediction error near the object edges directional prediction is also applied. Two directional predictors $\hat{a}_{\mathrm{h}}(\mathrm{i}, \mathrm{j})$ and $\hat{a}_{\mathrm{v}}(\mathrm{I}, \mathrm{j})$ are used for each pixel. These are calculated as follow:

$$
\begin{aligned}
& \hat{a}_{h}(i, j)=a_{o}(i, j-1) \\
& \hat{a}_{v}(i, j)=\operatorname{round}\left(a_{e}(i, j)+a_{e}(i+1, j)\right) / 2
\end{aligned}
$$

One of these is selected to predict a(i,j). For the most pixels the vertical predictor is used because it is used in both past and future of image signal.

Algorithm 1: Calculation of $\hat{a}_{\mathrm{o}}(\mathrm{i}, \mathrm{j})$ if $\operatorname{dir}(\mathrm{i}-1, \mathrm{j})=\mathrm{H}$ or $\operatorname{dir}(\mathrm{i}, \mathrm{j}-1)=\mathrm{H}$ then

Calculate dir $(\mathrm{i}, \mathrm{j})$

Encode dir (i, j) if $\operatorname{dir}(\mathrm{i}, \mathrm{j})=\mathrm{H}$ then

else $\hat{a}_{\mathrm{o}}(\mathrm{i}, \mathrm{j}) \leftarrow \hat{\mathrm{a}}_{\mathrm{h}}(\mathrm{i}, \mathrm{j})$

$\hat{a}_{o}(i, j) \leftarrow \hat{a}_{v}(i, j)$ end if

else

$\hat{a}_{o}(i, j) \leftarrow \hat{a}_{v}(i, j)$

Calculate dir $(\mathrm{i}, \mathrm{j})$ 
The calculation of the predictors is described in the Algorithm 1. Here $\mathrm{V}$ and $\mathrm{H}$ mean the vertical and horizontal edge directions. dir $(i, j)$ is $\mathrm{H}$ only when the current pixel $a_{0}(i, j)$ is near to the horizontal estimate $\hat{a}_{h}(i, j)$ than the vertical estimate $\hat{a}_{v}(i, j)$. In algorithm 2 the parameter $T_{1}$ is introduced where $\left|a_{0}(i, j)-\hat{a}_{h}(i, j)\right|$ is very smaller than $\left|a_{0}(i, j)-\hat{a}_{v}(i, j)\right|$. The calculation of dir $(i, j)$ is described in algorithm2.

Algorithm 2: Calculation of dir (i, j) if $\left|a_{o}(i, j)-\hat{a}_{h}(i, j)\right|+T_{1}<\left|a_{o}(i, j)-\hat{a}_{v}(i, j)\right|$ then
$\operatorname{dir}(i, j) \leftarrow H$

else

end if

$$
\operatorname{dir}(i, j) \leftarrow V
$$

\section{PROPOSED METHOD}

Firstly, an input RGB color image is transformed into $\mathrm{YCuCv}$ color space by improved reversible color transform as described in Section II. To encode the luminance image $\mathrm{Y}$ the standard lossless image coder, such as CALIC is used. The chrominance images $\mathrm{Cu}$ and $\mathrm{Cv}$ are encoded using the method as described Section III.

For efficient compression, the prediction error $e(i, j)=a_{o}$ $(i, j)-\hat{a}_{0}(i, j)$ is modeled with the coding context $C_{i}$ which is defined as below:

$\mathrm{C}_{\mathrm{i}}=\left\{(\mathrm{y}, \mathrm{x}): \mathrm{q}_{\mathrm{i}-1} \leq \alpha(\mathrm{y}, \mathrm{x})<\mathrm{q}_{\mathrm{i}}\right\}, \quad 1 \leq \mathrm{i} \leq \mathrm{K}$

here $\mathrm{q}_{\mathrm{o}}=0$ and $\mathrm{q}_{\mathrm{k}}=\infty$.

At pixel $\mathrm{a}_{\mathrm{o}}(\mathrm{x}, \mathrm{y})$ the representation of the local activity $\alpha$ (y, $\mathrm{x})$ is as below:

$\alpha(y, x)=\left|a_{e}(y, x)-a_{e}(y+1, x)\right|$

In all the experiments number of context $\mathrm{K}$ and the parameter $T_{1}$ in the Algorithm 2 are set as 6 and 3. For each context, the prediction errors are encoded by the modified Huffman coder $[13,14]$.

The major steps of our proposed method for image compression summarizing the following steps:

1) Select an input color images.

2) RGB color image is transfer into $\mathrm{YCuCv}$ color space by improved reversible color transform (RCT).

3) CALIC is used to encode the luminance image $Y$.

4) The chrominance images $\mathrm{Cu}$ and $\mathrm{Cv}$ encode by hierarchical decomposition and directional prediction method as below:

i) The chrominance channel of image is separated row by row into two subimages- an even subimage $\mathrm{A}_{\mathrm{e}}$ and odd subimage $\mathrm{A}_{\mathrm{o}}$.

ii) The prediction and encoding of the odd subimage $\mathrm{A}_{\mathrm{o}}{ }^{(1)}$ is occurred using the $\mathrm{A}_{\mathrm{e}}^{(1)}$ subimage.

iii) The even subimage $A_{e}^{(1)}$ is further decomposed column by column into even subimage $\mathrm{A}_{\mathrm{e}}^{(2)}$ and odd subimage $\mathrm{A}_{\mathrm{o}}{ }^{(2)}$.

iv) Using $A_{e}^{(2)}$ the $A_{o}^{(2)}$ is compressed.
5) The prediction error is calculated.

6) The prediction error is modeled with coding context.

7) For each context, a modified Huffman coder is used to encode the prediction error.

The proposed method in the form of flow chart is given below:

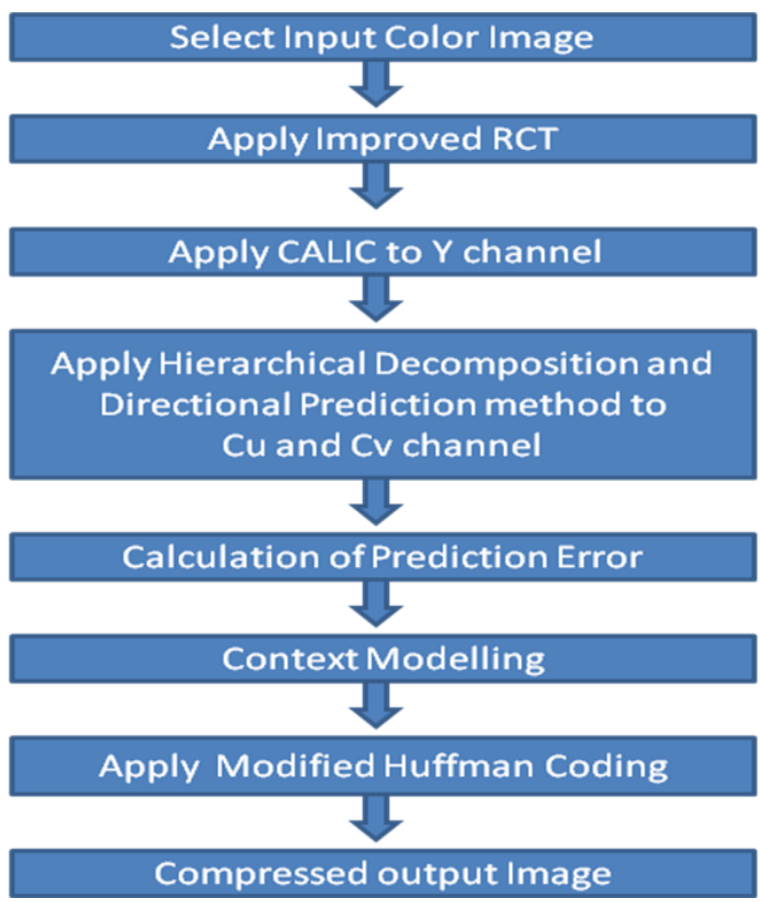

Fig - 3: Flow chart of proposed method

\section{EXPERIMENTAL RESULTS}

In the introduction already stated that the lossless image compression method may be the CALIC [4], which shows higher coding gain than the JPEG-LS (or LOCO-I) [2] [3] at the cost of higher computational complexity. Recently, the hierarchical prediction and context adaptive coding (HPCAC) method [12] shows the better result than the previous lossless image compression methods. Here the comparison will be made between the existing hierarchical prediction and context adaptive (HPCAC) method with our proposed scheme. As for test images [15], we have taken 08 medical images, 08 commercial digital camera images and 04 classic images. For comparison, bit per pixels (bpp) are measured for the compression streams.

As medical image we have taken the following images as shown in Fig. 4

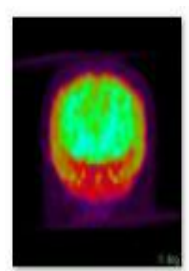

[0_pet]

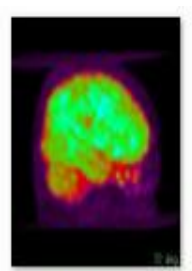

[0.pet2



[0_pet3

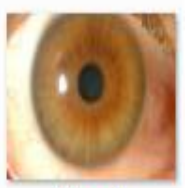

[1_eyel 


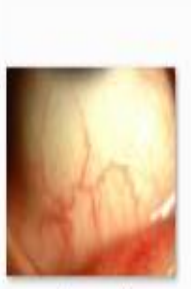

[1. evel

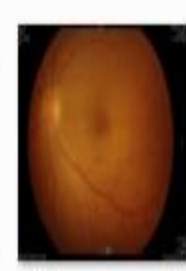

[2 eveeground



endoscopel

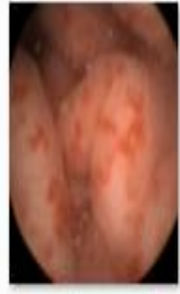

endoscope2
Fig - 4: The medical images

The Table-I shows the compressed bit rates (bpp) for the medical images.

Table 1: Compressed Bit Rates (bpp) for Medical Images

\begin{tabular}{|l|l|l|l|}
\hline Image & Size & $\begin{array}{l}\text { Previous } \\
\text { HPCAC } \\
\text { method }\end{array}$ & $\begin{array}{l}\text { Proposed } \\
\text { method }\end{array}$ \\
\hline PET1 & $256 \times 256$ & 5.6453 & 4.3994 \\
PET2 & $256 \times 256$ & 6.1598 & 4.7451 \\
PET3 & $256 \times 256$ & 5.8768 & 4.5889 \\
Eye1 & $3216 \times 2136$ & 4.6208 & 2.0670 \\
Eye2 & $3216 \times 2136$ & 4.3350 & 1.8994 \\
Eyeground & $1600 \times 1216$ & 2.9656 & 2.8888 \\
Endoscope1 & $608 \times 552$ & 7.0451 & 5.7238 \\
Endoscope2 & $568 \times 506$ & 4.8968 & 3.9060 \\
\hline Average & 5.1932 & 3.7773 \\
\hline
\end{tabular}

Fig. 5 shows the comparison of bits per pixel (bpp) of medical images for previous hierarchical prediction and context adaptive coding (HPCAC) method and our proposed method.

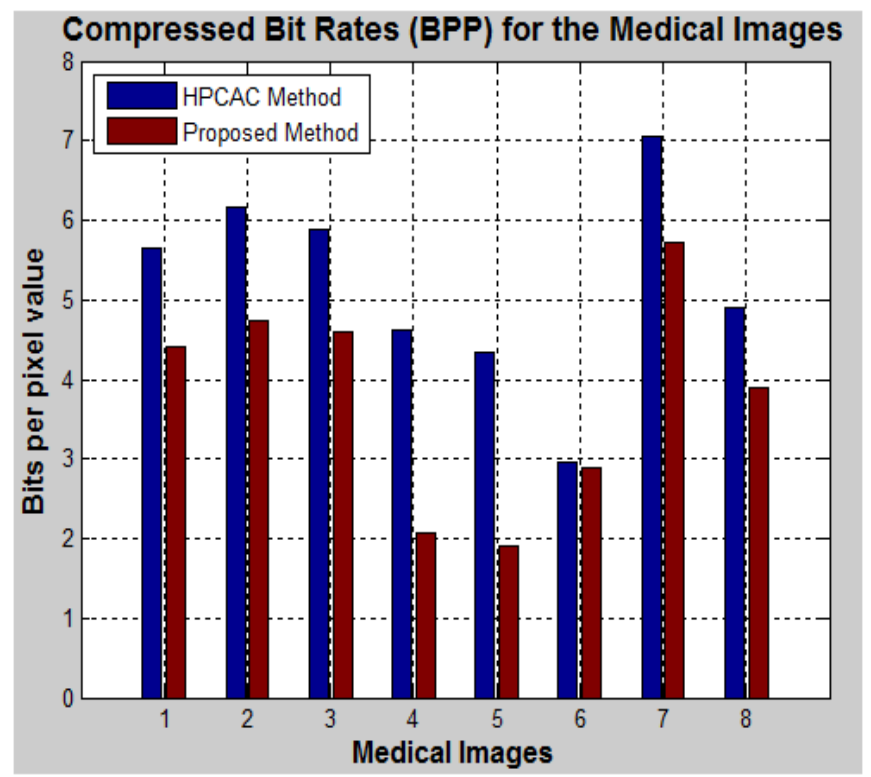

Fig - 5: Compressed bit rates for Medical Images

On the average the proposed algorithm produce $27.26 \%$ less bits than the existing method.

As commercial digital camera images we have taken the following images as shown in Fig. 6

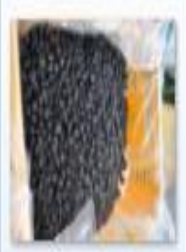

berly

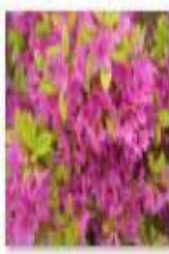

flower

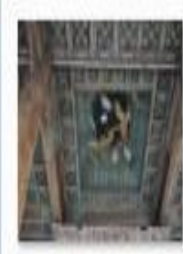

ceiling



locks

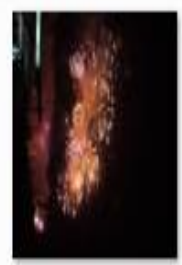

fireworks

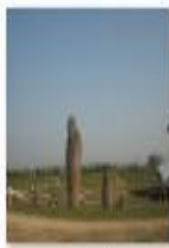

park

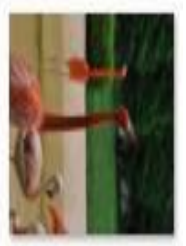

flamingo



sunset
Fig - 6: The digital camera images

The Table-II shows the bit per pixels for the commercial digital camera images:

Table 2: Compressed Bit Rates (bpp) for Commercial Digital Camera Images

\begin{tabular}{|l|l|l|l|}
\hline Image & Size & $\begin{array}{l}\text { Previous HPCAC } \\
\text { method }\end{array}$ & $\begin{array}{l}\text { Proposed } \\
\text { method }\end{array}$ \\
\hline Ceiling & $4288 \times 2848$ & 7.2080 & 5.4668 \\
Locks & $4288 \times 2848$ & 7.1623 & 6.0402 \\
Flamingo & $4288 \times 2848$ & 6.6371 & 2.7518 \\
Berry & $4288 \times 2848$ & 6.8917 & 5.6239 \\
Sunset & $4288 \times 2848$ & 5.9700 & 3.4576 \\
Flower & $4032 \times 3024$ & 6.0655 & 4.5139 \\
Park & $4032 \times 3024$ & 5.5622 & 3.4905 \\
Fireworks & $4032 \times 3024$ & 5.2855 & 2.2319 \\
\hline Average & 6.3478 & 4.1971 \\
\hline
\end{tabular}

Fig. 7 shows the comparison of bits per pixel (bpp) of digital camera images for previous hierarchical prediction and context adaptive coding (HPCAC) method and our proposed method.

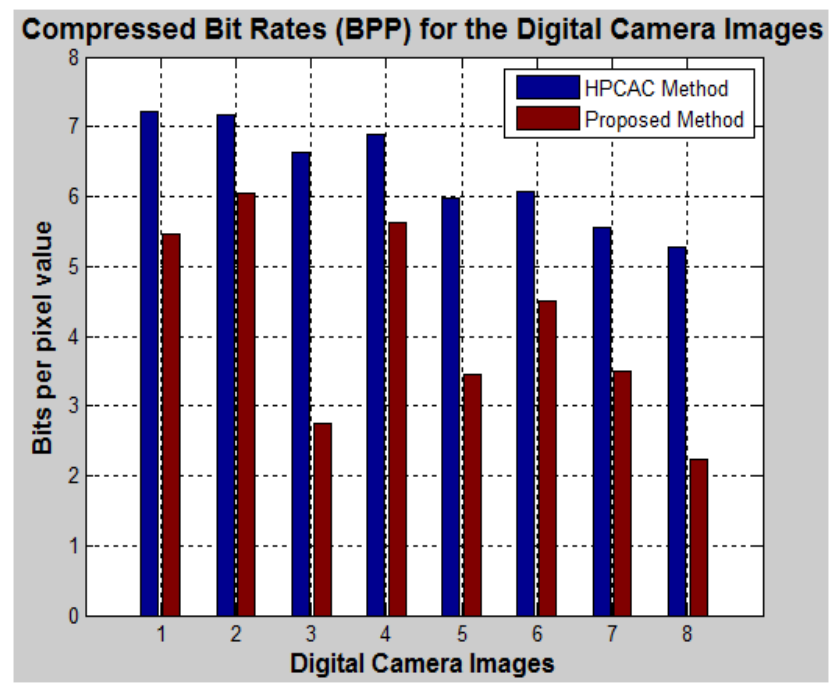

Fig- 7: Compressed bit rates for Digital Camera Images 
On the average the proposed algorithm produce $33.88 \%$ less bits than the existing method.

As classic images we have taken the following images as shown in Fig. 8

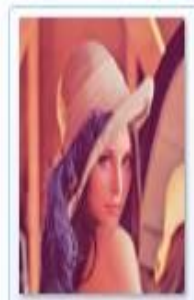

0 lena

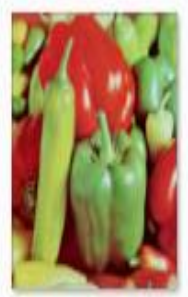

1_peppers

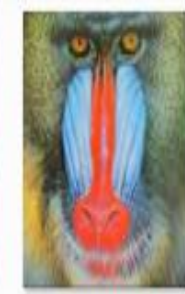

2 mandrill

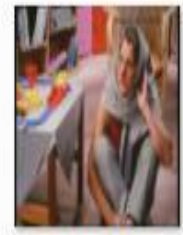

3 barbara
Fig- 8: The classic images

The Table- 3 shows the compressed bit rates (bpp) for the classic images:

Table 3: Compressed Bit Rates (bpp) for the Classic Images

\begin{tabular}{|l|l|l|l|}
\hline Image & Size & $\begin{array}{l}\text { Previous HPCAC } \\
\text { method }\end{array}$ & $\begin{array}{l}\text { Proposed } \\
\text { method }\end{array}$ \\
\hline Lena & $512 \times 512$ & 13.6461 & 8.9790 \\
Peppers & $512 \times 512$ & 15.2102 & 9.4900 \\
Mandrill & $512 \times 512$ & 18.5305 & 19.6958 \\
Barbara & $512 \times 512$ & 11.4575 & 10.9527 \\
\hline Average & 14.7111 & 12.2794 \\
\hline
\end{tabular}

Fig. 9 shows the comparison of bits per pixel (bpp) of classic images for previous hierarchical prediction and context adaptive coding (HPCAC) method and our proposed method.

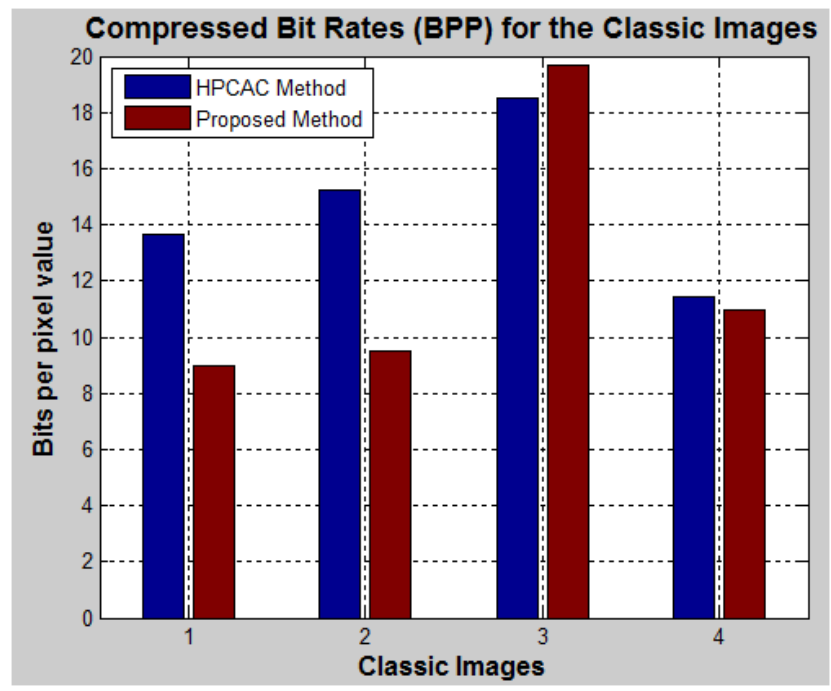

Fig- 9: Compressed bit rates for Classic Images

On the average the proposed algorithm produce $16.53 \%$ less bits than the existing method.

Fig. 9 shows the comparison of average bits per pixel (bpp) of medical images, digital camera images and classic images for previous hierarchical prediction and context adaptive coding (HPCAC) method and our proposed method.

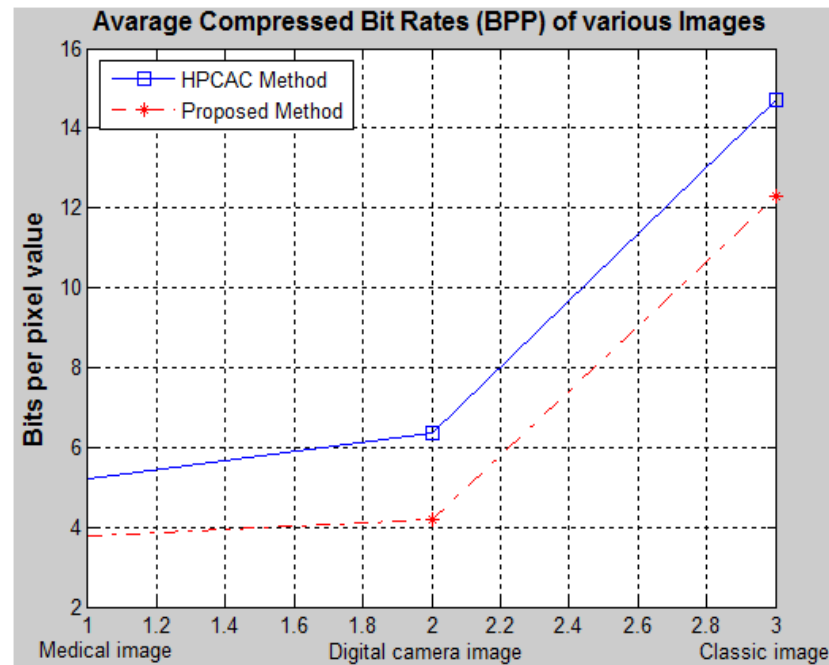

Fig - 10: Average compressed bit rates for various images

Finally, it is seen that than the previous hierarchical prediction and context adaptive coding (HPCAC) method our proposed method performed better.

\section{CONCLUSION}

Here we have proposed a lossless color image compression base on the hierarchical prediction and modified Huffman coding. Firstly, we transform the R, G and B color space of an input image into $\mathrm{YCuCv}$ color space using improved reversible color transform. The improved reversible color transform increase the de-correlation performance. After the color transform the luminance channel $\mathrm{Y}$ is compressed by a conventional lossless image coder. The chrominance channel is encoded with proposed hierarchical decomposition and directional prediction method and finally context modeling with modified Huffman coding is used. It is seen that by the proposed method the performance of compressed bit rates (bpp) for medical images, commercial digital camera image and classic image is improved by $27.26 \%, 33.88 \%$ and $16.53 \%$.

\section{REFERENCES}

[1]. Information Technology-Lossless and Near-Lossless Compression of Continuous-Tone Still Images (JPEGLS), ISO/IEC Standard 14495-1, (1999).

[2]. Pennebaker, W. B. and Mitchell, J. L., "JPEG Still Image Data Compression Standard", New York: Van Nostrand Reinhold, (1993).

[3]. M. Weinberger, G. Seroussi, and G. Sapiro, "The LOCO-I lossless image compression algorithm: principles and standardization into JPEG-LS," IEEE Trans. On Image Proc., vol. 9, no. 8 (2000), 1309-1324.

[4]. X. Wu and N. Memon, "Context-based, adaptive, lossless image coding," IEEE Trans. Commun., vol. 45 (1997), 437 - 444.

[5]. ITU-T and ISO/IEC, JPEG XR Image Coding SystemPart 2: Image Coding Specification, ISO/IEC Standard 29199-2 (2011). 
[6]. Information Technology_JPEG 2000 Image Coding System-Part 1: Core Coding System, INCITS/ISO/IEC Standard 15444-1 (2000).

[7]. $\mathrm{X}$. Wu and N. Memon, "Context-based lossless interband compression-Extending CALIC," IEEE Trans. Image Processing, vol. 9 (2000). 994-1001.

[8]. Enrico Magli, Gabriella Olmo, Emanuele Quacchio, "Optimized on-board lossless and near-lossless compression of hyperspectral data using CALIC," IEEE Geoscience and Remote Sensing Letters, Vol.1, No.1 (2004), 21-25.

[9]. Malvar, H.S., Sullivan, G.J., and Srinivasan, S., "Lifting-based reversible color transformations for image compression," Proc. SPIE Applications of Digital Image Processing XXXI, San Diego, CA, USA, Vol. 7073 (2008), 707307.1-707307.10.

[10]. Seyun Kim and Nam Ik Cho, "A lossless color image compression method based on a new reversible color transform", Visual Communications and Image Processing (VCIP) IEEE, (2012), 27-30.

[11]. P. Roos, M. A. Viergever, M. C. A. van Dijke, and J. H. Peters, "Reversible intraframe compression of medical images," IEEE Trans. Med. Imag., vol. 7, no. 4, (1988), 328-336.

[12]. Seyun Kim and Nam Ik Cho, "Hierarchical Prediction and Context Adaptive Coding for Lossless Color Image Compression", IEEE Transaction on Image Processing, Vol. 23, No. 1, (2014).

[13]. Donald L. Duttweiler and Christodoulos Chamzas, "Probability Estimation in Arithmetic and AdaptiveHuffman Entropy Coders", IEEE TRANSACTIONS ON IMAGE PROCESSING, VOL. 4, NO. 3.(1995).

[14]. MING-I LU and CHANG-FUU CHEN, "An Encoding Procedure and a Decoding Procedure for a New Modified Huffman Code", IEEE TRANSACTIONS ON ACOUSTICS. SPEECH, AND SIGNAL PROCESSING. VOL. 38, NO. I , (1990)

[15]. (Dec. 3, 2013) [Online]. Available: http://ispl.snu.ac.kr/light4u/project/ LCIC 